\title{
Integrated control of seed maturation and germination programs by activator and repressor functions of Viviparous-1 of maize
}

\author{
Ute Hoecker, ${ }^{1}$ Indra K. Vasil, and Donald R. McCarty ${ }^{2}$ \\ Plant Molecular and Cellular Biology Program, Department of Horticultural Sciences, University of Florida, Gainesville, \\ Florida 32611 USA
}

\begin{abstract}
The Viviparous-1 (VP1) transcriptional activator of maize is required for abscisic acid induction of maturation-specific genes late in seed development leading to acquisition of desiccation tolerance and arrest in embryo growth. Here, we show that VP1 also inhibits induction of the germination-specific $\alpha$-amylase genes in aleurone cells of the developing seed and thereby appears to be involved in preventing precocious hydrolyzation of storage compounds accumulating in the endosperm. In developing seeds of the somatically instable $v p 1-m 2$ mutant, hydrolase activity was derepressed specifically in endosperm sectors underlying $v p 1$ mutant aleurone. A barley $\alpha$-amylase promoter-GUS reporter construct (Amy-GUS) was induced in developing $v p 1$ mutant aleurone cells but not in wild-type aleurone cells. Moreover, transient expression of recombinant VP1 in vp1 mutant aleurone cells strongly inhibited expression of Amy-GUS and thus effectively complemented this aspect of the mutant phenotype. VP1 specifically repressed induction of Amy-GUS by the hormone gibberellic acid in aleurone of germinating barley seeds. Deletion of the acidic transcriptional activation domain of VP1 did not affect the inhibitory activity, indicating that VP1 has a discrete repressor function. Hence, physically combining activator and repressor functions in one protein may provide a mechanism to integrate the control of two normally consecutive developmental programs, seed maturation and seed germination.
\end{abstract}

[Key Words: Maize; Viviparous-1 gene; $\alpha$-amylase genes; seed maturation; seed germination]

Received June 5, 1995; revised version accepted August 30, 1995.

The formation of seeds is a unique characteristic of higher plants that promotes dispersal of the species and allows interruption of the life cycle during unfavorable environmental conditions. To survive in the dry state, plant embryos undergo an adaptation process during late stages of seed formation (maturation phase) that renders them tolerant to desiccation and gradually causes arrest in growth. In maize and other cereals, the outermost layer of the seed endosperm (aleurone layer) also undergoes a maturation process and remains viable through desiccation.

Seed maturation is associated with the activation of a variety of genes encoding storage proteins and various hydrophilic, late-embryogenesis-abundant (LEA) proteins that possibly function as desiccation protectants (Dure et al. 1989; Skriver and Mundy 1990). Analysis of viviparous mutants in maize has demonstrated that the developmental program of seed maturation is controlled

\footnotetext{
${ }^{1}$ Present address: U.S. Department of Agriculture, Plant Gene Expression Center, Albany, California 94710 USA.

${ }^{2}$ Corresponding author.
}

by at least two factors, the hormone abscisic acid (ABA) and the product of the Viviparous-1 $(V p 1)$ gene (Robertson 1955; Neill et al. 1986). Developing vp1 mutant embryos are distinct from ABA-deficient embryos in that they exhibit a reduced sensitivity to ABA (Robichaud et al. 1980; Robichaud and Sussex 1986). In addition to causing vivipary, the $v p 1$ mutation blocks synthesis of anthocyanins in embryo and aleurone tissues (Robertson 1955; Dooner 1985).

The Vp1 gene was cloned and shown to be expressed specifically in the developing seed (McCarty et al. 1989a). It encodes a novel protein with a functional acidic transcriptional activation domain (McCarty et al. 1991). Overexpression of VP1 in maize protoplasts transactivated reporter constructs containing late-embryogenesis-specific promoters: $C 1$, a maize gene that encodes a transcription factor required for anthocyanin synthesis in the seed, and Em, a wheat LEA gene (McCarty et al. 1991; Hattori et al. 1992). In agreement with the phenotype of ABA-deficient mutants, VP1 activation of $E m$ was strongly dependent on the presence of exogenous ABA (McCarty et al. 1991). 
In rehydrated cereal seeds, the germination-specific $\alpha$-amylase genes that encode starch-hydrolyzing enzymes are induced in the aleurone cells by the hormone gibberellic acid (GA) that is secreted by the embryo early in germination (Jacobsen and Chandler 1987). They are constitutively expressed in degermed seeds of the barley GA-response mutant slender /Chandler 1988; Lanahan and Ho 1988/, and their induction can be antagonistically inhibited by application of ABA (Jacobsen and Chandler 1987).

Expression of the seed maturation and seed germination programs is under strict developmental control. Precocious induction of germination-related events prior to seed maturity appears to be actively repressed. In the developing seed, $\alpha$-amylase activity is absent prior to seed maturity (Wilson et al. 1973; Evans et al. 1975). Moreover, $\alpha$-amylase genes are unresponsive to applied GA (Nicholls 1979; Cornford et al. 1986; Garcia-Maya et al. 1990; Oishi and Bewley 1990). It has been suggested that the presence of $\mathrm{ABA}$ in developing seeds is responsible for the inhibition of $\alpha$-amylase genes at this developmental stage (King 1976). However, treatment of developing maize seeds with the ABA synthesis inhibitor flouridone was not sufficient to sensitize the aleurone cells to GA, suggesting the action of additional factors in repressing $\alpha$-amylase genes in the developing seed /Oishi and Bewley 1990).

The advanced stages of viviparous seed development in the $v p 1$ mutant resemble normal seed germination. In particular, if vivipary is not interrupted by desiccation of the seed, the starchy endosperm is eventually digested and absorbed by the embryo. A corresponding sharp increase in $\alpha$-amylase enzyme activity is detected in the vp1 mutant endosperm relative to wild type beginning during the final quarter of normal seed formation (Wilson et al. 1973). However, the primary effects of the $v p 1$ mutant on maturation-related gene expression and anthocyanin synthesis are detected relatively early in development (McCarty et al. 1989a, 1991), it has been unclear whether $V p 1$ has a direct role in inhibiting germination-specific gene expression or, alternatively, whether the germination program eventually initiates by default in the absence of seed maturation. Insight into this question and, consequently, the role of the Vp1 gene in coordinating expression of the maturation and germination programs has come from analysis of genetically mosaic endosperms generated by a somatically unstable mutant allele of $v p 1$.

In this paper we show that in mosaic endosperms derepression of hydrolase activity is highly localized to $v p 1$ mutant sectors of the aleurone. We show that transient expression of recombinant VP1 in vp1 mutant aleurone strongly inhibits expression of an $\alpha$-amylase promoterGUS reporter gene (Amy-GUS). We present evidence that VP1 specifically represses GA induction of AmyGUS in aleurone of germinating barley seeds. In addition, we show that repression does not require the activator domain of VP1 or the presence of ABA, indicating that VP1 has a discrete function in a repression mechanism that is independent of ABA antagonism of GA ac- tion. Thus, we suggest that the coupled activator and repressor functions of VP1 play a key role in integrating control of the maturation and germination programs in seed development.

\section{Results}

The aleurone germination response is derepressed in developing vpl mutant kernels

The $v p 1-m 2$ allele of $V p 1$ carries a transposon insertion in the third intron that causes somatic instability of the gene during endosperm development (McCarty et al. 1989 b). As a result, mosaic kernels develop with clonal vp1 mutant and wild-type sectors. In these kernels, a striking pattern of endosperm remobilization is often evident. Endosperm tissue underlying $v p 1$ mutant aleurone cells is softened and depressed in surface, whereas wildtype sectors are raised relative to adjacent mutant sectors. This produces kernels with a distinctive etched appearance (Fig. 1A). The softening response was also observed when only a small fraction of the endosperm was

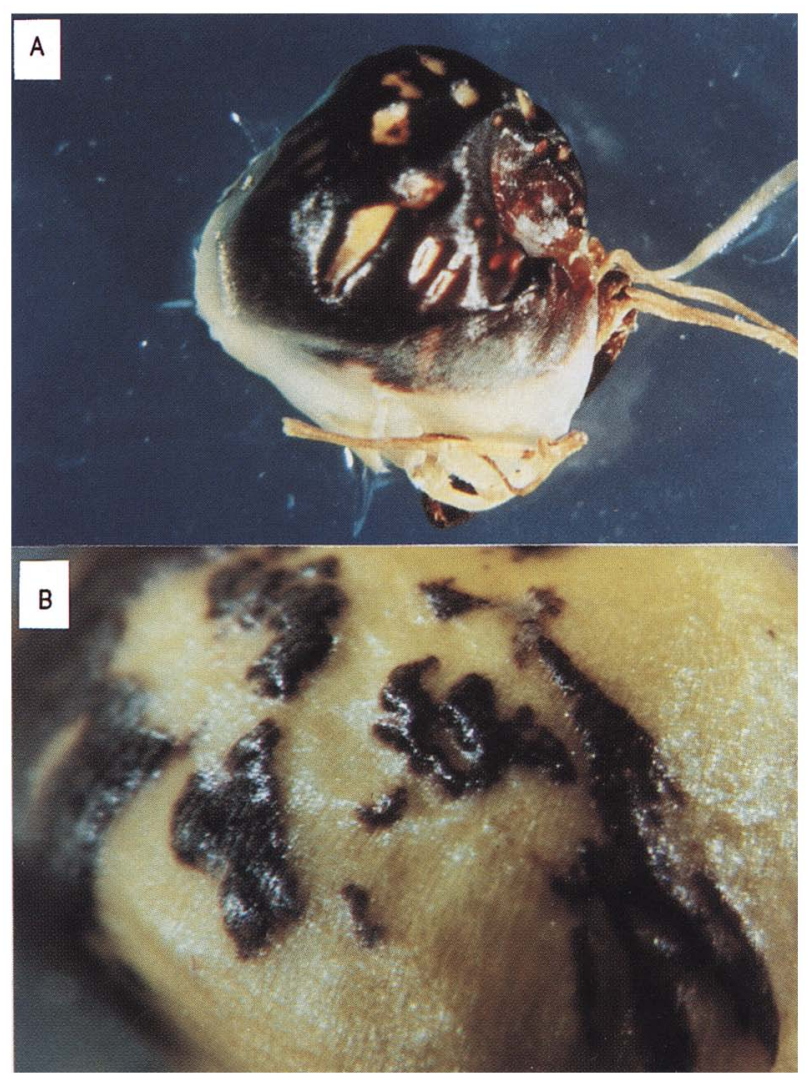

Figure 1. Highly localized derepression of the aleurone germination response in $v p 1-m 2$ mutant aleurone sectors. (A) The kernel shown is a mosaic: Regions pigmented with purple anthocyanin are wild-type; yellow anthocyanin-deficient regions are clonal sectors of aleurone that have lost $V p 1$ function. (B) Magnification of a $v p 1-m 2 \mathrm{kernel}$ normal sectors with a mutant background. 
comprised of normal tissue (Fig. 1B) indicating that this phenotype is nearly cell autonomous. We attribute the highly localized hydrolysis of the starchy endosperm underlying islands of $v p 1$ mutant aleurone cells to precocious induction and secretion of germination-specific hydrolytic enzymes in cells that have lost VP1 function. The sloped edges, which affect a halo of cells that are adjacent to the sector boundaries marked by the strictly cell-autonomous anthocyanin phenotype (McCarty et al. 1989a), are consistent with the expected diffusion of the secreted hydrolytic enzymes. This implies strongly that like the anthocyanin pathway, the repression of the germination-specific hydrolase genes is cell autonomously controlled by VP1.

The differential response of mutant and wild-type aleurone cells may not be fully independent of the state of the embryo. The embryos of $v p 1-m 2$ seed are frequently nonviviparous and survive desiccation. We have observed that mosaic aleurones that are associated with nonviviparous embryos rarely exhibit precocious endosperm remobilization, whereas those with viviparous embryos typically do. Moreover, aleurone near the germinal face and crown of the kernel is more strongly affected than aleurone on the abgerminal face (Fig. 1A).

To more directly address the role of VP1 in repressing hydrolase activity, a quantitative transient gene expression assay was developed based on particle bombardment of aleurone tissue with a barley high-pI Amy-GUS. We first determined whether the differential activity of hydrolases in vp1 mutant and wild-type sectors was the result of transcriptional control. For this purpose, AmyGUS was introduced into aleurone cells of developing vp1 mutant and wild-type maize seeds. Table 1 shows that throughout development Amy-GUS was not expressed in developing wild-type aleurone, even in the presence of exogenous GA. In contrast, GA induction of Amy-GUS was detected in $v p 1$ mutant aleurones as early as 20 days after pollination (DAP). These data indicate that in developing wild-type aleurone tissue, $\alpha$-amylase genes are insensitive to GA, whereas in $v p 1$ mutant aleurone cells, $\alpha$-amylase expression is transcriptionally active.

Amy-GUS expression in $v p 1$ mutant aleurone was found to be under developmental as well as hormonal control (Table 1). Prior to 18 DAP, Amy-GUS was inactive in GA-treated as well as untreated aleurone, indicating that early in seed development the aleurone is unresponsive to GA even in the absence of VP1 protein. At 20 DAP, Amy-GUS was induced by exogenous GA, whereas its activity remained low in untreated aleurones. Late in seed development $(\geqslant 24$ DAP), Amy-GUS was constitutively active in the absence of GA, indicating a reduced dependence on exogenous hormone. The onset of GA-independent Amy-GUS expression during the final quarter of ear development is in good agreement with the late onset of $\alpha$-amylase activity accumulation in the vp1 mutant (Wilson et al. 1973). However, this time frame does not exclude the possibility that additional post-transcriptional controls affect the timing of $\alpha$-amylase synthesis. Although, without hormone treatment Amy-GUS expression was consistently active in mutant aleurones late in development, the relative effects of exogenous GA treatment varied among experiments from no significant effect (Table 1) to a threefold enhancement over untreated aleurones (e.g., Fig. 2). We cannot rule out the possibility that variations in endogenous GA levels in developing viviparous seed contribute to the high variation in Amy-GUS activity we detect in the absence of exogenous GA.

\section{VP1 mediates trans-repression of $\alpha$-amylase expression in maize aleurone}

The differential expression of Amy-GUS in developing vp1 mutant and wild-type aleurone cells suggests a role of VP1 in the repression of $\alpha$-amylase genes. To test whether expression of recombinant VP1 could evoke repression of $\alpha$-amylase transcription in vp1 mutant aleurones, aleurones were bombarded with a mixture of Amy-GUS and 35S-Sh-VP1 plasmids. Coexpression of VP1 strongly inhibited Amy-GUS expression in vp1 mutant aleurone in the presence as well as absence of exogenous GA (Fig. 2), indicating that recombinant VP1 effectively restored the wild-type phenotype. We can rule out the possibility that overexpression of VP1 causes nonspecific squelching of general transcription factors because no inhibitory effect on 35S-Sh-GUS or ubiquitin-luciferase expression was observed /data not

Table 1. Amy-GUS is inducible in vp1-R mutant aleurone cells but not in wild-type aleurone cells

\begin{tabular}{|c|c|c|c|c|c|c|c|c|}
\hline \multirow{4}{*}{$\begin{array}{l}\text { Days after } \\
\text { pollination }\end{array}$} & \multicolumn{8}{|c|}{ Amy-GUS/LUC [pmoles MU/hr/10 $10^{4}$ RLU] } \\
\hline & \multicolumn{6}{|c|}{$v p 1-R$ mutant aleurones } & \multicolumn{2}{|c|}{ wild-type aleurones } \\
\hline & \multicolumn{3}{|c|}{$-\mathrm{GA}$} & \multicolumn{3}{|c|}{$+\mathrm{GA}$} & \multirow{2}{*}{$\frac{-\mathrm{GA}}{\text { mean }}$} & \multirow{2}{*}{$\frac{+\mathrm{GA}}{\text { mean }}$} \\
\hline & range & mean & S.E.M. & range & mean & S.E.M. & & \\
\hline 18 & & $<1$ & & & $<1$ & & $<1$ & $<1$ \\
\hline 20 & & $<1$ & & 4-35 & 21 & \pm 7 & $<1$ & $<1$ \\
\hline 24 & $114-531$ & 263 & \pm 130 & $41-150$ & 110 & \pm 35 & $<1$ & $<1$ \\
\hline
\end{tabular}

Aleurones of developing $v p 1-R$ mutant and wild-type kernels at 18,20, and 24 DAP were bombarded with a mixture of $10 \mu g$ of Amy-GUS and $5 \mu \mathrm{g}$ of Ubi-LUC. Following bombardment, kernels were treated with a solution containing no hormones or $10^{-6} \mathrm{M}$ $\mathrm{GA}_{3}$. All data represent range or mean (IS.E.M.) of three to five replicates. 


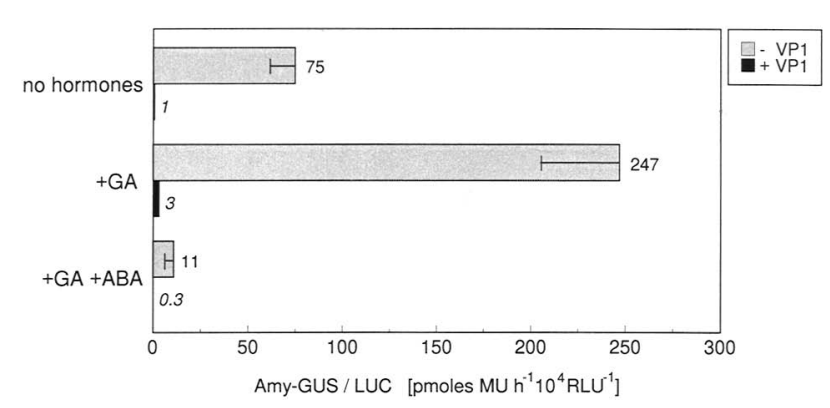

Figure 2. Effect of VP1 overexpression and ABA on Amy-GUS expression in $v p 1-R$ mutant aleurone. $v p 1-R$ mutant aleurones from kernels harvested 26 DAP were bombarded with $10 \mu \mathrm{g}$ of Amy-GUS, $5 \mu \mathrm{g}$ of Ubi-LUC, and $5 \mu \mathrm{g}$ of 35S-Sh-VP1 or 35SSh-CAT (for no-VPl controls). Following bombardment, a solution containing no hormones, $10^{-6} \mathrm{M} \mathrm{GA}_{3}$ or $10^{-6} \mathrm{M} \mathrm{GA}_{3}$ and $10^{-4} \mathrm{M} \mathrm{ABA}$ was applied to the kernels. The numbers to the right of the bars represent means of four replicates. Error bars show S.E.M.

shown). Moreover, as expected, VP1 caused trans-activation of positively regulated reporter constructs, EmGUS and C1-Sh-GUS, in aleurone cells using similar bombardment conditions $(S$. Cocciolone and D.R. McCarty, unpubl.; see below).

\section{VP1 and ABA function independently in repressing Amy-GUS}

In concert with VP1, the hormone ABA plays an important role during seed maturation (McCarty and Carson 1991). Moreover, ABA functions as an inhibitor of $\alpha$-amylase expression in germinating cereal seeds (Jacobsen and Chandler 1987). This suggests that ABA might also be involved in repression of $\alpha$-amylase genes in the developing seed. We were therefore interested in analyzing possible interactions between $\mathrm{ABA}$ and VP1 in repressing Amy-GUS.

Figure 2 shows that $\mathrm{ABA}$ was effective in blocking Amy-GUS expression in vp1 mutant aleurone indicating that inhibition by ABA does not require VP1. To test the converse, whether $\alpha$-amylase repression by VP1 is dependent on ABA, recombinant VP1 was expressed in aleurone of developing $v p 1$, vp5 double mutant kernels that are deficient for ABA biosynthesis. Figure 3 shows that VP1 was highly effective in repressing Amy-GUS in vp5 mutant background. Although we cannot rule out the possibility that maternal ABA derived from the $v p 5 /+$ parent plant is sufficient for VP1 function, we suggest that VP1-mediated repression of Amy-GUS expression does not require ABA. This is also consistent with the finding that VPl also functions in aleurone of germinating seeds (see below) where ABA levels are very low (Oishi and Bewley 1990). Taken together, these data indicate that ABA and VP1 inhibit Amy-GUS expression independently.

VP1 mediates repression in aleurone of germinating seeds of maize and barley

Endogenous expression of $V p 1$ in embryo and aleurone

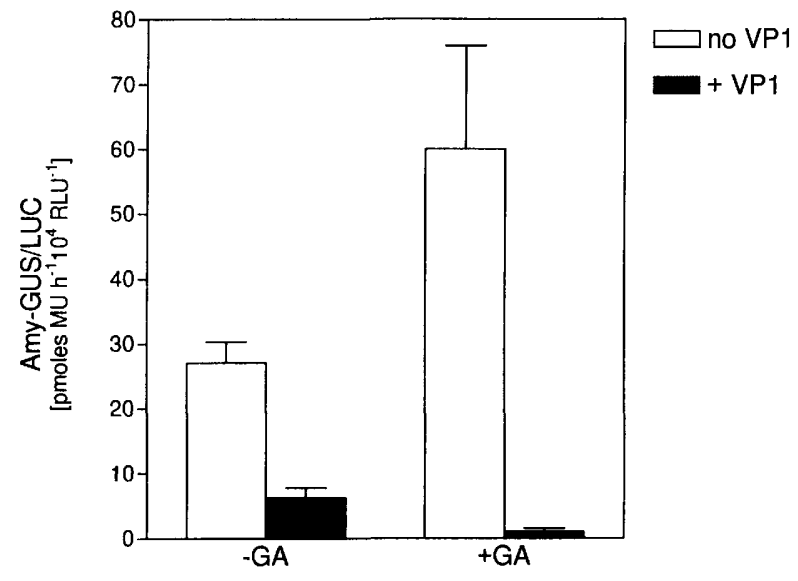

Figure 3. Coexpressed VP1 inhibited Amy-GUS in aleurone of developing $v p 1, v p 5$ double mutant seeds that are deficient for ABA biosynthesis. Kernels were harvested 24 DAP. Bombardments were performed as described in Fig. 2. Following bombardment, kernels were incubated in no hormones or $10^{-6} \mathrm{M}$ $\mathrm{GA}_{3}$. Data represent mean (IS.E.M.) of seven to eight replicates.

tissues is under developmental control. Vp1 mRNA peaks at $16 \mathrm{DAP}$ and then gradually decreases as the seed reaches maturity (McCarty et al. 1991). Germinating seeds, in contrast, display no $V p 1$ expression or detectable levels of VP1 protein (Carson 1993). Thus, VP1 function in maize is limited to the maturing seed. To test whether VPl can function in germinating seeds as well as in maturing seeds, we coexpressed $35 \mathrm{~S}-\mathrm{Sh}-\mathrm{VP} 1$ and Amy-GUS in aleurones of germinating wild-type seeds of maize. In the presence of exogenous GA, VP1 reduced Amy-GUS expression by $\sim 95 \%$ (Table 2 ). Thus, VP1 also functions in germinating seeds, apparently without the need for additional developmental factors.

Because germination-specific responses are well characterized in barley, we tested for VP1-mediated repression in aleurones of germinating barley seeds. Though not as effective as in maize, VP1 also inhibited AmyGUS expression in barley (Table 2). Overall, VP1 repressed Amy-GUS by a range of $60 \%-85 \%$ (data not shown).

To confirm that the inhibitory effect of VPl on Amy-

Table 2. Coexpressed VP1 inhibited Amy-GUS in aleurone of germinating maize and barley seeds

\begin{tabular}{lcc}
\hline & \multicolumn{2}{c}{ Amy-GUS/LUC } \\
\cline { 2 - 3 } Effector construct & maize & barley \\
\hline 35S-Sh-CAT (control) & $118( \pm 11)$ & $1556( \pm 299)$ \\
35S-Sh-VP1 & $8( \pm 1.2)$ & $240( \pm 30)$ \\
\hline
\end{tabular}

Aleurones of imbibed seeds were bombarded with Amy-GUS (maize, $5 \mu \mathrm{g}$; barley, $2 \mu \mathrm{g}$ ), $5 \mu \mathrm{g}$ of Ubi-LUC, and $5 \mu \mathrm{g}$ of 35SSh-VPl or 35S-Sh-CAT. Following bombardment, kernels were incubated in $10^{-6} \mathrm{M} \mathrm{GA}_{3}$. Data represent means (IS.E.M.) of three to five replicates. 
GUS in barley aleurone is promoter specific, 35S-ShVPl was coexpressed with an Em-GUS reporter construct containing the full-length promoter of the wheat $\mathrm{Em}$ gene fused to the GUS coding sequence (Marcotte et al. 1989|. Recombinant VPl increased expression of EmGUS by approximately sixfold (Fig. 4), which is consistent with the function of VP1 as a transcriptional activator of Em (McCarty et al. 1991). The gene activation and repression responses were detected over a similar range of VP1 dosage. Em-GUS was activated at 35S-ShVP1 doses as low as $1 \mu \mathrm{g}$ of plasmid DNA. In comparable experiments, $1.25 \mu \mathrm{g}$ of 35S-Sh1-VP1 effector caused 49 $( \pm 7) \%$ repression of the GA-induced Amy-GUS activity in barley aleurones, or $\sim 60 \%$ of the full response. A $5-\mu \mathrm{g}$ dosage of effector DNA achieved $>90 \%$ of the maximum Amy-GUS repression response obtained in barley and maize aleurones (Table 2; data not shown). In conclusion, these data show that VPl can cause either activation or repression of transcription in aleurone cells depending on the target promoter.

\section{VP1 blocks GA induction of $\alpha$-amylase genes}

The well-characterized hormonal responses in Himalaya barley aleurone facilitated further studies regarding the interaction between VPl and GA. For this purpose, GA response curves of Amy-GUS expression were determined in aleurones of degermed imbibed Himalaya "half seeds" (Fig. 5). In the absence of coexpressed VP1, AmyGUS expression showed a typical GA induction. In contrast, when a mixture of Amy-GUS and recombinant VPl was introduced into aleurone cells, GA induction of Amy-GUS expression was reduced by $\sim 80 \%$. The clearly detectable basal activity of Amy-GUS was not significantly affected by coexpression of VP1. Thus, VP1 inhibited only the GA-dependent activity of the $\alpha$-amylase promoter in barley.

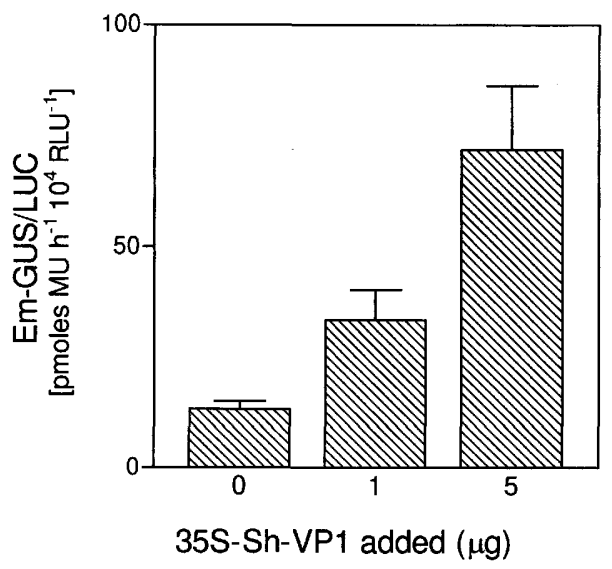

Figure 4. Coexpression of VP1 activated Em-GUS in barley aleurone. Aleurones of germinating barley seeds were bombarded with $2 \mu \mathrm{g}$ of Em-GUS, $5 \mu \mathrm{g}$ of Ubi-LUC, and $5 \mu \mathrm{g}$ of $35 \mathrm{~S}-\mathrm{Sh}-\mathrm{VP1}$ or $35 \mathrm{~S}-\mathrm{Sh}-\mathrm{CAT}$. Following bombardment, endosperms were cultured in no hormones. Data represent mean $( \pm \mathrm{SEM})$ of five replicates.

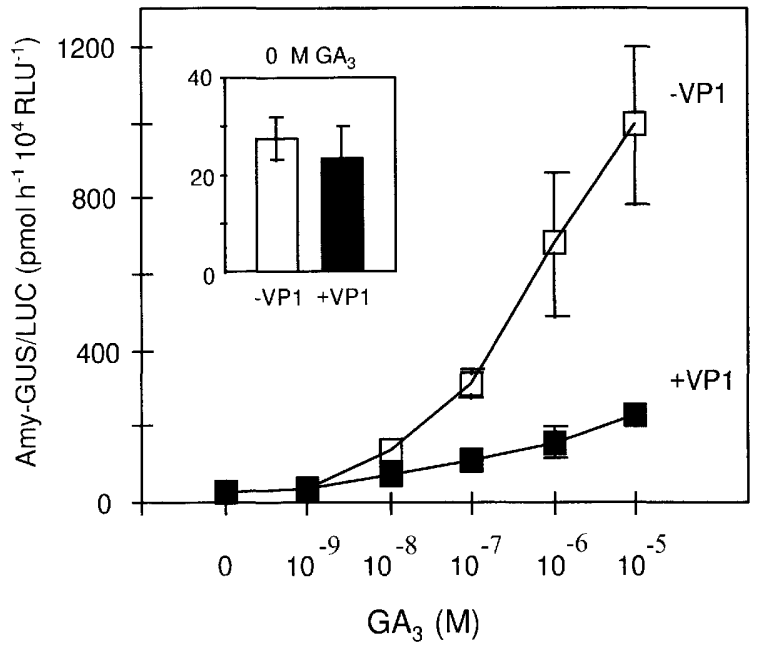

Figure 5. Coexpression of VPl inhibited $\mathrm{GA}_{3}$ induction of Amy-GUS but did not affect basal activity of the promoter in aleurone of germinating barley half seeds. Aleurones were bombarded with $4 \mu \mathrm{g}$ of Amy-GUS, $5 \mu \mathrm{g}$ of Ubi-LUC, and $5 \mu \mathrm{g}$ of $35 \mathrm{~S}-\mathrm{Sh}-\mathrm{VP1}$ or $35 \mathrm{~S}-\mathrm{Sh}-\mathrm{CAT}$. Following bombardment, three replicates of five kernels each were incubated in $0-10^{-5} \mathrm{M} \mathrm{GA}_{3}$. Data represent mean of three replicates ( \pm S.E.M). The insert shows activities in the absence of $\mathrm{GA}_{3}$.

VP1 function does not require the product of the GA-response gene slender

Recessive mutations that cause a constitutive GA response have been identified in barley and a few other species (Ross 1994). slender (sIn) mutant plants of barley are characterized by excessive elongation of stem and leaf tissues and constitutive expression of hydrolytic enzymes in the aleurone of imbibed half seeds in the absence of exogenous GA. The mutant phenotype suggests that the $\operatorname{SIn}$ gene encodes a negative regulator that is normally inactivated by GA (Chandler 1988; Lanahan and Ho 1988).

To test whether VP1 inhibitory function depends on the presence of the SLN protein, aleurones of sln mutant half seeds that had been imbibed for $12 \mathrm{hr}$ were cobombarded with Amy-GUS and recombinant VP1. Figure 6 shows that VP1-mediated repression of Amy-GUS was as effective in $\sin$ mutant aleurones as in wild-type aleurones. This indicates that VP1 is likely to act downstream in, or independently of, the SLN pathway.

\section{$\alpha$-Amylase repression is a discrete function of VP1}

We considered two models of how VP1 may function in repression of the aleurone germination response: (1) VP1 might be a transcriptional activator of an intermediate repressor gene that in turn inhibits expression of $\alpha$-amylase genes (Fig. 7A) and (2) VP1 itself might function as a repressor of the $\alpha$-amylase genes or of an intermediate gene that is required for activation of the $\alpha$-amylase promoter (Fig. 7B). To distinguish between these models, we determined whether the transcriptional activation do- 


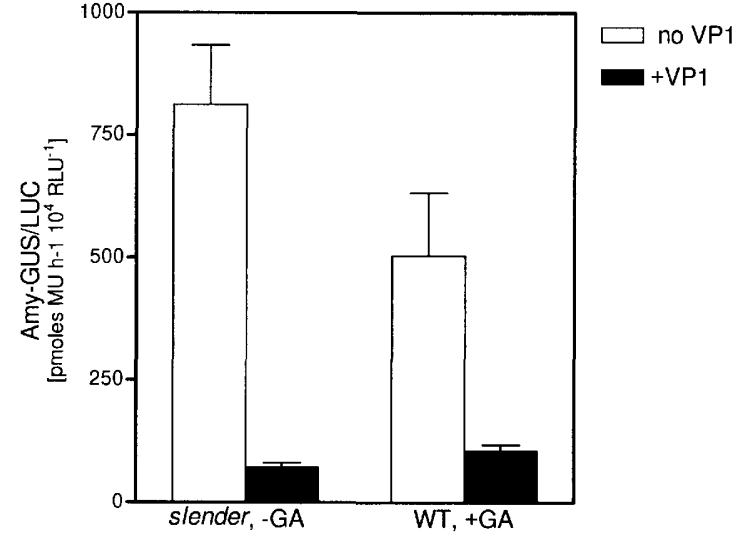

Figure 6. VP1 function does not require the $\operatorname{Sln}$ gene product. Aleurones of imbibed $\sin$ mutant and wild-type barley half seeds harvested from a plant segregating for the $\sin$ mutation were bombarded with plasmid DNA as described in Fig. 5. Following bombardment, sh mutant and wild-type seeds were incubated in a solution containing no hormones or $10^{-6} \mathrm{M} \mathrm{GA}_{3}$, respectively. Data represent mean ( \pm S.E.M) of 10 replicates.

main of VPl that is essential for activation of the $E m$ and C1 genes in maize cells is also required for inhibition of $\alpha$-amylase. Figure $7 \mathrm{C}$ shows that a deletion derivative of VP1 that lacks the anino-terminal activation domain was as effective in repressing Amy-GUS expression in maize and barley as the full-length protein. In addition, a VP16/VP1 hybrid protein that contains three copies of the acidic activation domain of the herpes simplex transcription factor VP16 and has a restored capacity to activate Em-GUS and Cl-Sh-GUS (McCarty et al. 1991; L. Rosenkrans and D.R. McCarty, unpubl.) was not more effective than the activator deletion mutant in causing repression of Amy-GUS. The lack of a requirement for the acidic activation sequence clearly distinguishes the mechanism of VP1-mediated repression from the mechanism of activation of diverse maturation-related genes by VP1. These results strongly indicate that the VP1 protein has a discrete repressor function.

\section{Analysis of VP1 deletion mutants}

Four mutant VP1 constructs containing deletions in the coding region were tested for their ability to repress Amy-GUS in maize and barley aleurone (Fig. 8). The $103 / 104$ and $86 / 85$ internal deletion mutants, respectively, had a slight effect $(<15 \%$ reduction $)$ and no effect, respectively, on the capacity of VP1 to repress AmyGUS in maize or barley aleurones. Similarly, truncation of the carboxy-terminal 155 amino acids of VP1 (VP1$\mathrm{McW}$ ) reduced repression by $27 \%$ in maize but did not significantly affect repression in barley. In contrast, the 137 -amino-acid deletion in $87 / 88$ caused $65 \%$ and $90 \%$ reductions in the repression of Amy-GUS in maize and barley aleurones, respectively, indicating that sequences in this region of VP1 are critical for the repressor function. In addition, the $87 / 88$ mutant caused an approxi- mately fivefold activation of Amy-GUS in barley aleurones in the absence of GA (Fig. 8). The gain of a novel activation function concomitant with loss of the repressor function of $87 / 88$ has at least two possible explanations: The mutant protein might act as a dominant negative by interacting with components of an endogenous repression mechanism in barley. Alternatively, it is possible that the mutant protein itself functions as an activator of Amy-GUS. In either case, although we have not ruled out quantitative effects of the $87 / 88$ on mRNA or protein stability, this result indicates that the $87 / 88$ protein retains a significant in vivo activity and that VP1 function is qualitatively altered by the mutant. Moreover, when tested in electroporated maize protoplasts (Hattori et al. 1992), the $87 / 88$ mutant activated the $\mathrm{Cl}$ Shl-GUS reporter gene $58( \pm 4) \%$ as effectively as wildtype VP1, indicating that the mutant also retains a significant capacity for activation of transcription in maize cells.

\section{Discussion}

VP1 of maize is a transcription factor that is specifically expressed in the developing seed (McCarty et al. 1989a, 1991). We have shown previously that VP1 is required for ABA-induced activation of a variety of genes associated
A

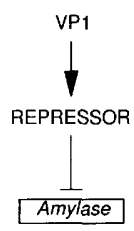

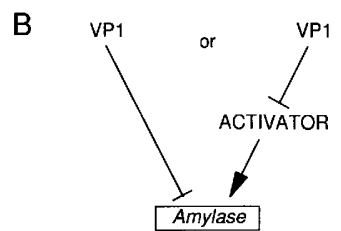

C
Figure 7. Mode of action of VPl in repressing Amy-GUS. $(A, B)$ Alternative models for VPl action as described in the text. $(C)$ Effect of an activation domain-deletion derivative of VP1 on Amy-GUS expression in aleurone tissue of germinating maize and barley seeds cultured in $\mathrm{GA}_{3}$. In the construct $\Delta 28-121$, the activation domain of VP1 was deleted. In $3 \times(\mathrm{VP1} 6 \mathrm{act})$, the activation domain of VPl was replaced by three copies of the acidic activation domain of the herpes simplex transcription factor VP16. Data represent activities (mean \pm S.E.M) relative to control $(=100)$. The VP16 activator domain is indicated by the solid area. The shaded areas represent regions of VP1 homology with $\mathrm{ABI} 3$. 


\begin{tabular}{|c|c|c|c|c|}
\hline & \multirow[t]{3}{*}{ Effector Construct } & \multicolumn{3}{|c|}{ Relative Amy-GUS activity. } \\
\hline & & \multirow{2}{*}{$\begin{array}{r}\text { Maize } \\
+ \text { GA }\end{array}$} & \multicolumn{2}{|c|}{ Barley } \\
\hline & & & $+\mathrm{GA}$ & $-\mathrm{GA}$ \\
\hline & 35S-Sh-CAT & $100^{( \pm 26)}$ & $10{ }^{( \pm 5.9)}$ & $5^{( \pm 1)}$ \\
\hline VP1-WT & CL & $8^{( \pm 2.5)}$ & $19^{( \pm 1.8)}$ & $5^{( \pm 1)}$ \\
\hline $86 / 85$ & $\square$ & $7^{( \pm 2)}$ & $13_{( \pm 2.7)}$ & $9( \pm 2)$ \\
\hline $87 / 88$ & प & $67^{( \pm 15)}$ & $90( \pm 19)$ & $24( \pm 2)$ \\
\hline 103/104 & 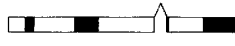 & $21( \pm 3.5)$ & $27^{( \pm 3)}$ & n.d. ${ }^{*}$ \\
\hline VP1-McW & $\square$ & $32( \pm 8.7)$ & $13^{( \pm 0.5)}$ & $3^{( \pm 1)}$ \\
\hline
\end{tabular}

Figure 8. Deletion analysis of the VP1 protein: Aleurones of maize and barley germinating seeds were bombarded with 2-5 $\mu \mathrm{g}$ of Amy-GUS, $5 \mu \mathrm{g}$ of Ubi-LUC, and $5 \mu \mathrm{g}$ of effector construct and then cultured in $10^{-6} \mathrm{MGA}_{3}$ or no $\mathrm{GA}_{3}$ as indicated. Data represent mean ( \pm S.E.M) of three to five replicates. (Solid areas) Sequence homology between VP1 and ABI3.

with seed maturation (McCarty et al. 1991). In this paper, we provide evidence that, in addition to its transcriptional activator function, VP1 has a specific role in blocking precocious induction of germination-specific $\alpha$-amylase genes during seed development.

\section{VP1 represses $\alpha$-amylase genes}

There are at least three lines of evidence that indicate a function of VP1 in repression of $\alpha$-amylase genes in the developing seed. First, somatically unstable vp1-m2 seeds displaying both $v p 1$-mutant and wild-type sectors showed highly localized derepression of endosperm remobilization specifically in sectors underlying $v p 1 \mathrm{mu}-$ tant aleurone (Fig. 1A,B). Second, in transient expression experiments, Amy-GUS was inducible or constitutively active in developing $v p 1$ mutant aleurone cells but not in wild-type aleurone cells (Table 1). Third, coexpression of recombinant VP1 with Amy-GUS in vp1 mutant aleurone cells inhibited Amy-GUS expression by $>95 \%$ (Fig. 2 ). Our results are consistent with findings that $\alpha$-amylase genes are not expressed in the developing seed (Nicholls 1979; Cornford et al. 1986; Garcia-Maya et al. 1990; Oishi and Bewley 1990). Hence, cessation of VP1 expression prior to germination may be necessary to allow induction of $\alpha$-amylase genes in the germinating seed.

Gene repression is distinct from activation functions of $V P 1$

In contrast to the mechanism of transcriptional activation of maturation-specific genes, VP1-mediated repres- sion of $\alpha$-amylase genes does not require the transcriptional activation function located at the amino-terminal domain of VP1 (Fig. 7). This indicates that VP1 has a function in repression that is mechanistically distinct from the transcriptional activation function. Several systems in which a single transcription factor functions as both an activator and a repressor depending on the promoter context have been described in animals (Miner and Yamamoto 1991; Tsai and O'Malley 1994). Direct structural homologs of VP1 are thus far known only in plants, suggesting that this strategy has evolved independently in plants and animals.

A series of internal deletion mutants delimit an internal region of the VP1 protein that is essential for repression activity. Interestingly, the regions of VP1 that are most highly conserved in the Arabidopsis ABI3 protein and other homologs cloned from plants (corresponding to the $86 / 85,103 / 104$, and VPl-McW deletions) are not essential for repression. The strong repression activity of the truncated VP1-McW protein is consistent with the nonviviparous, anthocyanin-deficient phenotype of the corresponding $v p 1-M c W$ allele in planta (McCarty et al. 1989b; Carson 1993). Thus, the highly conserved carboxy-terminal domain is necessary for activation of a class of maturation genes that includes the $C 1$ gene but not for prevention of germination. Our results do not delineate a specific molecular mechanism for VP1-mediated repression. The repression essential region of VP1 may not necessarily have an inherent repressor activity. One plausible function of this region that is consistent with several known mechanisms of repression would be to mediate specific protein-protein contacts with other factors. In that case, repression of transcription may be critically dependent on the context of the protein-protein interactions and not an inherent property of the sequence.

\section{Interactions between VP1 and plant hormones}

We can envision at least three models of how VP1 might function in repressing $\alpha$-amylase genes: (1) VP1 might mediate $\mathrm{ABA}$ antagonism of GA signaling during seed development. ABA is known to antagonize GA action in the regulation of $\alpha$-amylase genes in germinating cereal seeds (Jacobsen and Chandler 1987). Because VP1 is required for $\mathrm{ABA}$-induced gene expression associated with seed maturation (McCarty et al. 1991), it might also be essential in ABA-mediated repression of $\alpha$-amylase genes. Consequently, the $v p 1$ mutant might derepress $\alpha$-amylase genes because of a failure to respond to ABA present in the developing seed. (2) VP1 might specifically inhibit the GA response pathway independently of ABA. (3) VP1 might repress $\alpha$-amylase genes via a pathway that is independent of both GA and ABA signaling pathways.

Our results do not support the first model. ABA was effective in blocking Amy-GUS expression in vp1 mutant aleurone cells (Fig. 2), indicating that ABA action does not depend on the presence of VP1. This stands in contrast to evidence showing that VP1 is required for 
ABA-induced expression of the maize Em gene (McCarty et al. 1991). Thus, there appear to be at least two modes of ABA action in the maize seed, a VP1-dependent pathway and a VP1-independent pathway. Multiple ABA transduction pathways are also indicated by interactions between ABA-insensitive mutants of Arabidopsis (Finkelstein and Somerville 1990; Finkelstein 1994). This suggests that ABA modulates the activity of multiple regulatory cascades in the seed.

The second scenario in which VPl specifically blocks GA signal transduction is consistent with the evidence that overexpression of VP1 in aleurone of imbibed barley half seeds severely reduced GA induction of Amy-GUS without affecting the basal activity of the $\alpha$-amylase promoter (Fig. 5). This suggests that expression of VP1 in the developing seed may be, at least in part, responsible for the GA insensitivity of cereal and maize $\alpha$-amylase genes prior to seed maturity (Nicholls 1979; Cornford et al. 1986; Garcia-Maya et al. 1990; Oishi and Bewley 1990|. In our experiments, VP1 displayed full repressing activity in $\sin$ mutant barley seeds (Fig. 6) that are constitutive in GA response of the aleurone (Chandler 1988; Lanahan and Ho 1988), suggesting that VP1 functions at a point downstream of the $S I n$ gene product. The remaining formal possibility that $\operatorname{Sin}$ actually encodes the homologous VP1 function in barley is unlikely because $\sin$ affects GA responses in plant tissues as well as the seed but does not block seed maturation or other ABA responses. In contrast, the phenotypes of $v p 1$ and the homologous abi3 mutant of Arabidopsis (Giraudat et al. 1992; Parcy et al. 1994), a more distantly related species, are restricted to the maturation and germination phases of seed development.

With respect to the maize seed, our data do not rule out the possibility that VP1 acts independently of GA as a developmental repressor of a $\alpha$-amylase genes. Although we have shown that Amy-GUS is GA inducible in vp1 mutant aleurones early in development (Table 1), it is not clear that the high constitutive activities found later in development are entirely attributable to changes in GA concentration. In contrast to the situation of Himalaya barley seed and other cereal grains, studies of $\alpha$-amylase regulation in normal and GA-deficient (d5mutant/genotypes indicate that $\alpha$-amylase induction in germinating maize seeds is largely independent of GA (Harvey and Oaks 1974). Consistent with these studies, we also find that during germination Amy-GUS is constitutively active in the $\mathrm{GA}_{1}$-deficient $d 1$ mutant of maize (U. Hoecker, I.K. Vasil, and D.R. McCarty, unpubl.). Because Amy-GUS was fully VPl repressible in aleurones of developing vp1 mutant (Fig. 2), germinating wild-type (Table 2), and germinating $d 1$-mutant seeds (U. Hoecker, I.K. Vasil, and D.R. McCarty, unpubl.|, we suggest that VPl repression is not necessarily restricted to nor solely defined by inhibition of the GA signal. Rather, GA, ABA, and VP1 may be three among several factors that regulate the activity of constituents required for expression of $\alpha$-amylase genes. We suggest that maize and barley merely differ in the dependence of this seed-specific pathway on the presence of GA.
VP1 integrates the control of seed maturation and germination programs

We have shown that VP1 participates in the regulation of two developmental pathways in the developing maize seed. As a transcriptional activator it is required for activation of maturation-specific genes (McCarty et al. 1991), and as a repressor it prevents precocious induction of the normally germination-specific $\alpha$-amylase genes (this paper). Hence, expression of VPl specifically during seed development appears to be involved in ensuring proper ordering of maturation and germination programs. We suggest that physically combining activation and repression function in one protein provides one mechanism for directly integrating control of mutually exclusive developmental pathways in the plant embryo. The developmental importance of a tight control of maturation and germination programs for seed survival is evident in the phenotype of $v p 1-m 2$ seeds.

Premature induction of postgerminative development was also reported for the lec1 (leafy cotyledon 1) mutant of Arabidopsis (Meinke 1992). In this ABA-sensitive, viviparous mutant, developing embryos expressed isocitrate lyase genes and a gene encoding a lipid transfer protein at levels that are normally characteristic of seedlings (West et al. 1994). Double mutant analysis suggested that the putative Arabidopsis VP1 homolog, $\mathrm{ABI}$, and LEC1 function in different pathways (Meinke et al. 1994). Hence, it appears that multiple mechanisms have evolved in flowering plants to prevent precocious induction of normally germination-specific genes in the developing embryo.

Thus far, our evidence that VP1 inhibits germinationspecific genes is limited to hydrolase genes in aleurone cells. We do not know to what extent this repressor activity of VP1 is also involved in preventing precocious germination of the embryo. Further insight into the inhibitory role of VP1 during seed development may be provided by stable transformation of $v p 1$ mutant plants with VP1 derivatives that are mutated specifically in the activator or repressor functions.

Cloning of the Vp1 related genes from rice (Hattori et al. 1994), Arabidopsis (Giraudat et al. 1992), and tobacco (Phillips and Conrad 1994) indicates that the Vp1 gene is conserved among flowering plants. Loss of ABI3 function in Arabidopsis causes a viviparous phenotype similar to the vp1 mutation in maize (Nambara et al. 1992). The functions of $A B I 3$ and VP1, however, diverge in so far that $\mathrm{ABI} 3$ is required for seed dormancy in Arabidopsis whereas VP1 does not impose seed dormancy in maize. Because ABI3 mRNA is stored in the dry seed (Parcy et al. 1994), whereas VP1 transcript and protein are essentially absent in the mature seed (McCarty et al. 1989a; Carson 1993), we speculate that dormancy in Arabidopsis may reflect an extended timing of ABI3 expression after seed maturity rather than a functional difference in the proteins. This view is supported by our results showing that overexpression of VP1 in aleurone of germinating maize seeds was effective in repressing Amy-GUS (Table 2). A role of VP1 in maintaining seed dormancy is 
also consistent with the finding that dormancy in barley is correlated with a reduced GA inducibility of $\alpha$-amylase genes in the aleurone (Schuurink et al. 1992; Skadsen 1993). Hence, we suggest that VP1 plays a role in integrating the control of seed maturation, dormancy, and germination programs.

\section{Materials and methods}

Plant material

All developong maize seeds were harvested from field-grown plants. Under the local environmental conditions, kernels typically begin accumulation of anthocyanins at day 17 after pollination and reach seed maturity after $\sim 30-33$ DAP. The vp1-R allele (Robertson 1955) segregated in a color-converted W22 inbred line carrying all other factors required for anthocyanin pigmentation of the aleurone. This line is routinely maintained by selfing. The $v p 1-m 2$ allele (previously named $v p 1$-mum2; McCarty et al. 1989b/ arose in Robertson's Mutator transposable element stocks (Robertson 1978) but was confirmed to carry an Mpi transposable element insertion (D.R. McCarty, unpubl.). Seed segregating for the $v p 5$ mutation (and homozygous for recessive alleles of the $c 1 \mathrm{r} 1$ anthocyanin factors) was obtained from the Maize Genetics Corporation Stock Center (University of Illinois, Urbana-Champaign, IL). To produce vp1, $v p 5$ double mutant seeds, heterozygous vp5 plants were crossed to a fully pigmented W22 inbred stock $(\mathrm{A} 1, \mathrm{~A} 2, \mathrm{Bz}, \mathrm{Bz} 2, \mathrm{Cl}$, $\mathrm{R} 1)$. The anthocyanin factors were fixed in the $\mathrm{F} 3$ by selection of uniformly purple ears that also segregated $3: 1$ for the viviparous, carotenoid deficient vp5 phenotype. The resulting heterozygous $v p 5$ lines were crossed to heterozygous $v p 1-R$ plants that carried all other dominant factors $(\mathrm{A} 1, \mathrm{~A} 2, \mathrm{Bz} 1, \mathrm{Bz2}, \mathrm{Cl}, \mathrm{C} 2, \mathrm{R} 1)$ required for anthocyanin pigmentation. Double heterozygotes were identified by selection of F2 ears that segregated the following four classes: purple aleurone, yellow endosperm, nonviviparous embryo; purple aleurone, white endosperm, viviparous albino embryo; yellow aleurone, yellow endosperm, viviparous chlorophyllous embryo; white aleurone, white endosperm, viviparous albino embryo in a 9:3:3:1 ratio. Developing vp5, vp1 double mutant seed were recovered by self-pollination of double heterozygotes. Specifically, these were distinguished from the single mutant $v p 5$ class by the absence of anthocyanins in the aleurone and from the $v p 1$ single mutant class by the absence of carotenoids.

For experiments with germinating seeds of maize, seeds of the variety NK508 were used. Wild-type barley seeds c.v. Himalaya were obtained from Washington State University, Pullman, WA (harvest 1988). Seed segregating for the $\sin$ mutation (Himalaya background) was kindly provided by P. Chandler (CSIRO, Camberra, Australia). Genotypes of the wild-type and sin mutant endosperms were determined by germination of the excised embryo.

\section{Plasmid constructs}

In all experiments, Amy-GUS (JR254) was used as the reporter construct. This construct was kindly provided by J. Rogers (University of Missouri, Columbia). Amy-GUS contains $\sim 1800$ bp of the 5 -flanking sequence of a barley high-pI $\alpha$-amylase gene (Amy6-4; Khursheed and Rogers 1988), the first intron of Amy64, the GUS reporter gene and the Amy6-4 3' terminator. As effector construct, 35S-Sh-VPl was used (McCarty et al. 1991). For no-VP1 control treatments, 35S-Sh-CAT (Vasil et al. 1989) was added instead of $35 \mathrm{~S}-\mathrm{Sh}-\mathrm{VP} 1$ to maintain constant amounts of DNA and $35 \mathrm{~S}$ promoter in the bombardment mix- tures. To normalize for transformation efficiency, a ubiquitinluciferase construct (Ubi-LUC; Bruce et al. 1989) was included to each bombardment mixture. Cobombardment of 35S-ShVP1 exhibited no effect on expression of Ubi-LUC in the absence nor presence of GA.

Constructions of the activation domain deletion-derivative of VP1 $(\Delta 28-121)$ and the VP1/VP16 fusion derivative were described in McCarty et al. (1991). To generate internal deletions in the VP1 sequence, two NcoI restriction sites were introduced by site-directed mutagenesis using the Altered Sites in vitro Mutagenesis system from Promega. Following restriction of the mutant constructs with $\mathrm{NcoI}$, the insert was removed. The construct $86 / 85$ deletes amino acids 126-222. Constructs $87 / 88$ and $103 / 104$ delete amino acids $238-375$ or $387-404$, respectively. The VP1-McW construct is described in Carson (1993).

\section{Particle bombardment and transient expression assays}

Particle bombardment was performed as described in Taylor and Vasil (1991) using a DuPont PDS-1000 particle gun. DNA was precipitated onto $1.75 \mathrm{mg}$ of gold particles (BIO-Rad, 1.0 or $1.6 \mathrm{~nm}$ diam.), and $\sim 80 \mu \mathrm{g}$ of gold was used for individual bombardments. The protoplast electroporation assays used to test $87 / 88$ activation of $\mathrm{C} 1-\mathrm{Sh} 1-\mathrm{GUS}$ were performed as described by Hattori et al. (1992) with modifications described by Rosenkrans et al (1995). The relative activity of the $87 / 88$ construct was determined from triplicate electroporations as a percentage of the net activation induced by the wild-type 35S-Sh1-VP1 construct.

Quantitative measurement of GUS activities was performed as described in Jefferson (1987). The background GUS activities of barley and maize aleurones were in the range of 0.5 to 2.0 pmoles MU/aleurone/hr. The raw GUS activities for nonrepressed treatments were typically $>100$-fold over background. For determination of luciferase activities, $10-\mu \mathrm{l}$ aliquots of the extract and $200 \mu \mathrm{l}$ of reaction buffer $(25 \mathrm{~mm}$ tricine at $\mathrm{pH} 7.8,15$ $\mathrm{mM} \mathrm{MgCl}, 5 \mathrm{~mm} \mathrm{ATP}, 0.05 \% \mathrm{BSA}$ ) were placed in cuvettes and immediately assayed using a Monolight 2010 luminometer. The luminometer automatically injects $100 \mu \mathrm{l}$ of $1 \mathrm{~mm}$ luciferin and then counts the emitted photons for $15 \mathrm{sec}$. The unit of LUC activity is Relative Light Unit (RLU). Because the resulting LUC values are relatively large numbers, the normalized GUS activities are reported per 10,000 RLUs.

\section{Transient expression in maize and barley aleurone}

Maize developing ears or dry, mature seeds were surface sterilized in $70 \%$ ethanol for 1 min followed by $0.525 \% \mathrm{NaOCl}$ for 10 min. Dry seeds were germinated in a solution containing MS salts and MS vitamins (Sigma, cat no. M5519) on a gyratory shaker until the stage of radical emergence from the pericarp $(\sim 36 \mathrm{hr})$. Developing seeds were used immediately. Embryo as well as pericarp and testa were removed to expose the aleurone layer of the endosperm. The exposed endosperms were placed on Gelrite-solidified salt medium and subjected to particle bombardment. Following bombardment, $1 \mathrm{ml}$ of a solution containing MS salts and MS vitamins supplemented with no hormones, $10^{-6} \mathrm{M} \mathrm{GA}_{3}$ or $10^{-6} \mathrm{M} \mathrm{GA}_{3}$ and $10^{-4} \mathrm{M} \mathrm{ABA}$ was dripped over the endosperms. After $24 \mathrm{hr}$ of incubation in darkness, endosperms were ground either individually (in experiments using developing seeds) or in bulk from each bombardment (when germinating seeds were used) with mortar and pestle in 200 $1000 \mu \mathrm{l}$ of extraction buffer $(0.1 \mathrm{M}$ potassium phosphate at $\mathrm{pH}$ 7.8, 2 mM EDTA at pH 8, 2 mM DTT, 5\% glycerol/. The homogenates were centrifuged, and the supernatants were used for determination of GUS and LUC activities. 
Barley seeds were deembryonated, surface sterilized in $70 \%$ ethanol for $1 \mathrm{~min}$ followed by $10 \mathrm{~min}$ in $1.75 \% \mathrm{NaOCl}$, and then imbibed overnight and prepared for particle bombardment as described above for maize seeds. Twenty-four hours after bombardment, aleurone tissue was separated from the endosperm and ground in bulk for each replicate in $250 \mu \mathrm{l}$ of extraction buffer.

\section{Acknowledgments}

We thank John Rogers for providing Amy-GUS (JR254) and Leonard Rosenkrans for assistance with C1-Sh-GUS activation assays. This work was supported by a grant from the National Science Foundation (DCB-93-01414) to D.R.M and the U.S. Department of Agriculture (92122115) to D.R.M. and I.K.V. This paper is Florida Agriculture Experiment Station Journal series no. R04705

The publication costs of this article were defrayed in part by payment of page charges. This article must therefore be hereby marked "advertisement" in accordance with 18 USC section 1734 solely to indicate this fact.

\section{References}

Bruce, W.B., A.H. Christensen, T. Klein, M. Fromm, and P.H. Quail. 1989. Photoregulation of a phytochrome gene promoter from oat transferred into rice by particle bombardment. Proc. Natl. Acad. Sci. 86: 9692-9696.

Carson, C.B. 1993. "Characterization of the structure and expression of the mutant alleles of the viviparous-1 gene of maize." Ph.D. thesis, University of Florida, FL.

Chandler, P.M. 1988. Hormonal regulation of gene expression in the "slender" mutant of barley (Hordeum vulgare L.). Planta 175: 115-120.

Cornford, C.A., M. Black, J.M. Chapman, and D.C. Baulcombe. 1986. Expression of $\alpha$-amylase and other gibberellin-regulated genes in aleurone tissue of developing wheat grains. Planta 169: 420-428.

Dooner, H.K. 1985. Viviparous-1 mutation in maize conditions pleiotropic enzyme deficiencies in the aleurone. Plant Physiol. 77: 486-488.

Dure III, L., M. Crouch, J. Harada, T.H.D. Ho, J. Mundy, R. Quatrano, T. Thomas, and Z.R. Sung. 1989. Common amino acid sequence domains among the LEA proteins of higher plants. Plant Mol. Biol. 12: 475-486.

Evans, M., M. Black, and J. Chapman. 1975. Induction of hormone sensitivity by dehydration is one positive role for drying in cereal seed. Nature 258: 144-145.

Finkelstein, R.R. 1994. Mutations at two new Arabidopsis ABA response loci are similar to the abi3 mutations. Plant $I$. 5: 765-771.

Finkelstein, R.R. and C. Somerville. 1990. Three classes of abscisic acid (ABA)-insensitive mutations of Arabidopsis define genes that control overlapping subsets of ABA responses. Plant Physiol. 94: 1172-1179.

Garcia-Maya, M., J.M. Chapman, and M. Black. 1990. Regulation of $\alpha$-amylase formation and gene expression in the developing wheat embryo. Planta 181: 296-303.

Giraudat, J., B.M. Hauge, C. Valon, J. Smalle, F. Parcy, and H. Goodman. 1992. Isolation of the Arabidopsis $A B I 3$ gene by positional cloning. Plant Cell 4: 1251-1261.

Harvey, B.M.R. and A. Oaks. 1974. The role of gibberellic acid in the hydrolysis of endosperm reserves in Zea mays. Planta 121: 67-74.

Hattori, T., V. Vasil, L. Rosenkrans, L.C. Hannah, D.R.
McCarty, and I.K. Vasil. 1992. The Viviparous-1 gene and abscisic acid activate the $C 1$ regulatory gene for anthocyanin biosynthesis during seed maturation in maize. Genes $\&$ Dev. 6: 609-618.

Hattori, T., T. Terada, and S.T. Hamasuna. 1994. Sequence and functional analyses of the rice gene homologous to the maize Vp1. Plant Mol. Biol. 24: 805-810.

Jacobsen, J.V. and P.M. Chandler. 1987. Gibberellin and abscisic acid in germinating cereals. In Plant hormones and their role in plant growth and development (ed. P.J. Davies), pp. 164193. Martinus Nijhoff, Dordrecht, The Netherlands.

Jefferson, R.A. 1987. Assaying chimeric genes in plants: The GUS gene fusion system. Plant Mol. Biol. Rep. 5: 387-405.

Khursheed, B. and J.C. Rogers. 1988. Barley $\alpha$-amylase genes: Quantitative comparison of steady-state mRNA levels from individual members of the two different families expressed in aleurone cells. J. Biol. Chem. 263: 18953-18960.

King, R.W. 1976. Abscisic acid in developing wheat grains and its relationship to grain growth and maturation. Planta 132: 43-51.

Lanahan, M.B. and T.H.D. Ho. 1988. Slender barley: A constitutive gibberellin-response mutant. Planta 175: 107-114.

Marcotte, W.R., Jr., S.H. Russell, and R.S. Quatrano. 1989 Abscisic acid-responsive sequences from the Em gene of wheat. Plant Cell 1: 969-976.

McCarty, D.R. and C.B. Carson. 1991. The molecular genetics of seed maturation in maize. Physiol. Plant 81: 267-272.

McCarty, D.R., C.B. Carson, P.S. Stinard, and D.S. Robertson. 1989a. Molecular analysis of viviparous-1: An abscisic acidinsensitive mutant of maize. Plant Cell 1: 523-532.

McCarty, D.R., C.B. Carson, M. Lazar, and S.C. Simonds. 1989b. Transposable element-induced mutations of the viviparous-1 gene in maize. Dev. Genet. 10: 473-481.

McCarty, D.R., T. Hattori, C.B. Carson, V. Vasil, M. Lazar, and I.K. Vasil. 1991. The Viviparous-1 developmental gene of maize encodes a novel transcriptional activator. Cell 66: 895-905.

Meinke, D.W. 1992. A homeotic mutant of Arabidopsis thaliana with leafy cotyledons. Science 258: 1647-1650.

Meinke, D.W., L.H. Franzmann, T.C. Nickle, and E.C. Yeung. 1994. Leafy cotyledon mutants of Arabidopsis. Plant Cell 6: $1049-1064$.

Miner, J.N. and K.R. Yamamoto. 1991. Regulatory crosstalk at composite response elements. Trends Biochem. Sci. 16: $423-426$.

Nambara, E., S. Naito, and P. McCourt. 1992. A mutant of Arabidopsis which is defective in seed development and storage protein accumulation is a new abi3 allele. Plant $f$. 2: 435-441.

Neill, S.J., R. Horgan, and A.D. Parry. 1986. The carotenoid and abscisic acid content of viviparous kernels and seedlings of Zea mays L. Planta 169: 87-96.

Nicholls, P.B. 1979. Induction of sensitivity to gibberellic acid in developing wheat caryopses: Effect of rate of desiccation. Aust. I. Plant Physiol. 6: 229-240.

Oishi, M.Y. and J.D. Bewley. 1990. Distinction between the responses of developing maize kernels to flouridone and desiccation in relation to germinability, $\alpha$-amylase activity, and abscisic acid content. Plant Physiol. 94: 592-598.

Parcy, F., C. Valon, M. Raynal, P. Gaubier-Comella, M. Delseny, and J. Giraudat. 1994. Regulation of gene expression programs during Arabidopsis seed development: Roles of the $A B I 3$ locus and of endogenous abscisic acid. Plant Cell 6: 1567-1582.

Phillips, J. and U. Conrad. 1994. Genomic sequences from Nicotiana tabacum homologous to the maize transcriptional 
activator gene Viviparous-1. I. Plant Physiol. 144: 760-761.

Robertson, D.S. 1955. The genetics of vivipary in maize. Genetics 40: 745-760.

- 1978. Characterization of a mutator system in maize. Mutation Res. 51: 21-28.

Robichaud, C.S. and I.M. Sussex. 1986. The response of viviparous-1 and wild-type embryos of Zea mays to culture in the presence of abscisic acid. J. Plant Physiol. 126: 235-242.

Robichaud, C.S., J. Wong, and I.M. Sussex. 1980. Control and growth of viviparous embryo mutants of maize by abscisic acid. Dev. Genet. 1: 325-330.

Rosenkrans, L., V. Vasil, I. K. Vasil, and D. R. McCarty. 1995. Functional analysis of a plant transcription factor using transient expression in maize protoplasts. In Methods in plant molecular biology (ed. P. Maliga, D.F. Klessig, A.R., Cashmore, W. Gruissem, and J.E. Varner), pp. 19-36. Cold Spring Harbor Laboratory Press, Cold Spring Harbor, New York.

Ross, J.J. 1994. Recent advances in the study of gibberellin mutants. Plant Growth Regul. 15: 193-206.

Schuurink, R.C., N.J.A. Sedee, and M. Wang. 1992. Dormancy of the barley grain is correlated with gibberellic acid responsiveness of the isolated aleurone layer. Plant Physiol. 100: $1834-1839$.

Skadsen, R.W. 1993. Aleurones from a barley with low $\alpha$-amylase activity become highly responsive to gibberellin when detached from the starchy endosperm. Plant Physiol. 102: 195-203.

Skriver, K. and J. Mundy. 1990. Gene expression in response to abscisic acid and osmotic stress. Plant Cell 2: 503-512.

Taylor, M.G. and I.K. Vasil. 1991. Histology of, and physical factors affecting transient GUS expression in pearl millet (Pennisetum glaucum (L.) R. Br.) embryos following microprojectile bombardment. Plant Cell Rep. 10: 120-125.

Tsai, M. and B.W. O'Malley. 1994. Molecular mechanisms of action of steroid/thyroid receptor superfamily members. Annu. Rev. Biochem. 63: 451-486.

Vasil, V., M. Clancy, R.J. Ferl, I.K. Vasil, and L.C. Hannah. 1989. Increased gene expression by the first intron of the maize sh1 locus in grass species. Plant Physiol. 91: 1575-1579.

West, M.A., K.M. Yee, J. Danao, J.L. Zimmerman, R.L. Fischer, R.B. Goldberg, and J.J. Harada. 1994. Leafy cotyledon 1 is an essential regulator of late embryogenesis and cotyledon identity in Arabidopsis. Plant Cell 6: 1731-1745.

Wilson, G.F., A.M. Rhodes, and D.B. Dickinson. 1973. Some physiological effects of viviparous genes vpl and vp5 on developing maize kernels. Plant Physiol. 52: 350-356. 


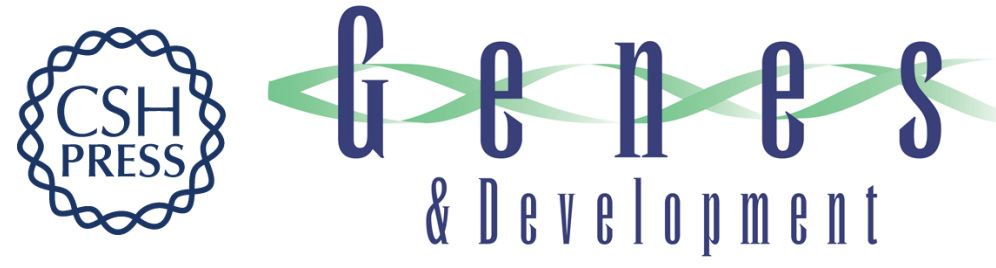

\section{Integrated control of seed maturation and germination programs by activator and repressor functions of Viviparous- 1 of maize.}

U Hoecker, I K Vasil and D R McCarty

Genes Dev. 1995, 9:

Access the most recent version at doi:10.1101/gad.9.20.2459

References This article cites 44 articles, 19 of which can be accessed free at:

http://genesdev.cshlp.org/content/9/20/2459.full.html\#ref-list-1

License

Email Alerting

Service

Receive free email alerts when new articles cite this article - sign up in the box at the top right corner of the article or click here.

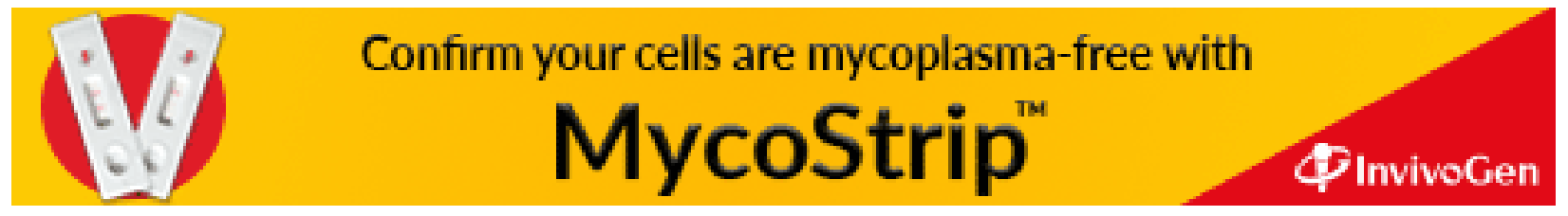

